\title{
Intervenções psicossociais no transtorno bipolar
}

\section{Psychosocial Interventions for Bipolar Disorder}

Luis Pereira Justo ${ }^{1}$

Helena Maria Calil ${ }^{2}$

\section{Resumo}

Neste trabalho, os autores, através de revisão bibliográfica narrativa, situam as intervenções psicossociais dentro do panorama terapêutico para o transtorno bipolar e constatam que ainda são insuficientes os estudos primários feitos com metodologia adequada para a obtenção de informações científicas de boa qualidade. São sucintamente descritos os trabalhos mais relevantes.

Palavras-chave : Transtorno bipolar, intervenções psicossociais, psicoeducação, psicoterapia, tratamento.

\section{Abstract}

In this paper, the authors review the status of psychosocial interventions within the general treatment for bipolar disorder. They have verified the scantiness of studies performed with adequate methodology to obtain scientific information of good quality. The more relevant studies are briefly described.

Keywords : Bipolar disorder, psychoeducation; psychosocial interventions, psychotherapy, treatment.

Recebido: 27/10/2003 - Aceito: 09/03/2004

1 Médico Psiquiatra, psicoterapeuta de Grupo-Análise pela SPAG-RJ, psicoterapeuta de Família pelo ITF-RJ, mestrando pela Universidade Federal de São Paulo-Escola Paulista de Medicina (Unifesp-EPM)

2 Professora titular de Psicofarmacologia, do Departamento de Psicobiologia da Universidade Federal de São Paulo-Escola Paulista de Medicina (Unifesp-EPM), pesquisadora IC, CNPq

Endereço para correspondência: Luis Pereira Justo. Depto de Psicobiologia da Universidade Federal de São Paulo (UNIFESP), Rua Napoleão de Barros, 925. 04024-002, São Paulo - SP, Brasil. Telefone: (11) 2888566, 99165596, e-mail: luisjusto1@ig.com.br 


\section{Introdução}

O diagnóstico do Transtorno Bipolar (TB), atualmente, pode ser feito a partir de critérios razoavelmente bem estabelecidos tanto na prática clínica quanto para efeito de pesquisa, embora seja ainda necessário o esclarecimento objetivo de aspectos cruciais como, por exemplo, a etiologia e o processo fisiopatológico. É considerado um transtorno crônico, caracterizado pela existência de episódios agudos, recorrentes, de alteração patológica do humor, com pelo menos um episódio de mania, hipomania ou misto. A recuperação após a fase aguda é geralmente significativa, mas menos completa e isenta de conseqüências da que seria desejável. Há estudos que demonstram a persistência de sintomas relacionados ao humor patológico, embora abrandados, em pacientes tratados com adequação sob o ponto de vista farmacológico (Muller-Oerlinghausen, 2000). Também é notável o impacto da experiência da doença sobre a personalidade do indivíduo acometido, levando a modificações e comumente perdas no modus vivendi, mesmo que os sintomas do humor propriamente ditos não sejam mais aparentes. Os critérios diagnósticos mais difundidos são os definidos pelo Manual Diagnóstico e Estatístico dos Transtornos Mentais - DSM-IV-R - que divide o TB em tipos I, II, transtorno ciclotímico e TB SOE -, e pela Classificação Internacional das Doenças, $10^{\mathrm{a}}$ edição (CID-10). Na primeira classificação, o tipo I é relacionado à presença de mania franca no mínimo em um episódio, podendo ou não ocorrer depressão ou episódios mistos; o tipo II diz respeito à presença de hipomania (desde que não tenha havido mania plena anteriormente), com a ocorrência de pelo menos um episódio de depressão maior; no transtorno ciclotímico há numerosos episódios de hipomania com muitos episódios de depressão que não preenchem critérios para depressão maior por pelo menos dois anos, sem intervalos maiores que dois meses livres de sintomas; e, finalmente, os pacientes que apresentam hipomania com sintomas depressivos não-classificáveis como portadores de depressão maior, com características de curso diferentes das modalidades anteriores, sendo incluídos nos TB SOE (APA, 2002). A CID-10 apresenta poucas diferenças substanciais em relação ao DSM-IV-R, apesar de excluir a ciclotimia dos transtornos afetivos bipolares, incluindo-a em transtornos persistentes do humor e não usar as designações I e II (CID-10, 1993). É possível identificar, na literatura, duas tendências divergentes: uma que valoriza a dis- tinção entre o TB e o Transtorno Unipolar (onde ocorrem somente episódios de depressão) (Goodwin e Jamison, 1990), e outra que alarga as fronteiras diagnósticas do TB, incluindo condições antes classificadas de outro modo, inclusive tentando recuperar a idéia de um continuum para os transtornos do humor, ou seja, esmaecendo a distinção bipolar/unipolar (Akiskal, 1996; Akiskal e Pinto, 1999). A prevalência do TB nos EUA varia entre $0,4 \%$ e $1,6 \%$ para o TBI e 0,5\% para o TB-II (APA, 2002). Não há diferenças relativas a gênero ou raça para a prevalência do TB I, mas o TB II parece ser mais freqüente nas mulheres (Kusumakar et al., 1997).

A morbidade, a mortalidade - com risco de suicídio 30 vezes maior do que para a população geral (Guze et al., 1970) -, o prejuízo da qualidade de vida, além dos custos elevados para o tratamento, fazem do TB um importante problema de saúde que demanda a maior otimização possível de manejo.

O uso de medicamentos, tais como lítio, anticonvulsivantes, antipsicóticos, antidepressivos, benzodiazepínicos, hormônios tireoideanos e, embora mais raramente, a aplicação da eletroconvulsoterapia, tornouse consenso como parte fundamental do tratamento, mesmo considerando-se o fato de não abarcarem todos os problemas inerentes ou concomitantes ao TB. É indispensável que o paciente reconheça seus sintomas, enquanto tais, e a necessidade de seu controle. Faz parte do trabalho terapêutico a informação ampla ao paciente, a respeito das características e vicissitudes de sua doença e das possibilidades de tratamento, visando ao maior grau possível de adesão. Cada vez mais, valoriza-se o uso de coadjuvantes, especialmente das intervenções chamadas de psicossociais, que incluem as diversas formas de psicoterapia e métodos de psicoeducação. Esses procedimentos podem exercer um papel de facilitação junto ao paciente para que ele participe do controle de sua doença, como também possibilitar o manejo das dificuldades criadas pela existência dela e que afetam sua vida de modo diverso, complexo e, muitas vezes, devastador (Goodwin e Jamison, 1990; Kusumakar et al., 1997).

\section{0 desafio de tratar}

Até a introdução do lítio e outros medicamentos na prática clínica, o TB seguia aproximadamente seu curso natural. As especulações sobre sua origem e tratamento eram variadas, incongruentes e geralmente de pouca utilidade prática. 
Entre as tentativas de tratamentos realizados, estavam as psicoterapias como, por exemplo, as de orientação psicodinâmica. Essas, acreditando na hipótese de psicogenia, investiam firmemente nas intervenções verbais como principal instrumento terapêutico. Logo foram notadas dificuldades ligadas ao comportamento próprio dos pacientes com TB, especialmente nos que apresentavam sintomas ativos de mania, e sugestões foram feitas de que tais pessoas necessitavam de formas mais específicas de terapia (Fromm Reichmann, 1949).

É compreensível que quadros agudos de mania, hipomania, depressão ou mistos e mesmo os sintomas residuais dos períodos interfásicos sem o uso de medicamentos eficazes se constituíssem empecilhos quase intransponíveis quando se oferecia ajuda psicoterápica a esses pacientes. Com o avanço da psicofarmacologia, a situação modificou-se a tal ponto, que o perfil do paciente também foi transformado, revelando acesso maior aos benefícios viabilizados pela palavra. No presente momento, parece haver a tendência à mudança de foco quando se administra uma psicoterapia, no sentido de se objetivar mais o manejo da doença do que sua possível causa, pois as evidências apontando para o predominante papel dos fatores orgânicos na etiologia têm sobrepujado as hipóteses de psicogenia.

Uma vez que os sintomas agudos tenham sido total ou parcialmente suprimidos, ganham importância fatores menos óbvios no princípio, mas não menos determinantes na morbidade da doença e nas dificuldades a serem enfrentadas pelo paciente, pessoas ligadas a ele e pelos terapeutas. Nota-se o prejuízo em capacidades cruciais como a de estabelecer e conservar laços afetivos com outras pessoas, devido às turbulências das oscilações do humor, e também dificuldades marcantes em se disponibilizar para o trabalho e produzir dentro dos padrões esperados. Nesse sentido, um estudo demonstrou que aproximadamente um terço dos pacientes permanecem incapacitados para o trabalho, e só $21 \%$ recuperam níveis anteriores de rendimento, seis meses após a alta hospitalar (Dion et al., 1988). A diminuição da auto-estima inerente à troca do status de pessoa saudável pelo de portadora de uma doença crônica, bem como os receios ligados à perda de controle de si próprio, alteram dramaticamente o modo de relação do paciente com o mundo e consigo mesmo.

Recuperar e manter a qualidade de vida prévia ao aparecimento do transtorno pode ser uma tarefa árdua e nem sempre inteiramente possível. Inicial- mente, há que se lidar com a problemática aceitação da existência do TB que tanto para o paciente quanto para a família tende a ser encarado como muitas coisas, exceto como uma doença que demanda cuidados onerosos sob múltiplos aspectos. A negação do problema é freqüente e um entrave ao tratamento. Além do mais, não basta estar ciente da existência do TB, mas é preciso compreender do modo mais amplo possível suas características e assumir a postura de co-responsável pelo tratamento, participando ativamente do estabelecimento de medidas protetoras. Talvez seja ainda mais delicada a necessidade de tolerar a impossibilidade de se chegar, pelo menos no momento atual, à cura do TB. Estudos longitudinais mostram que, mesmo com tratamento farmacológico apropriado, a taxa de recorrência em um ano pode chegar a $40 \%$ e, em cinco anos, a $73 \%$. Mesmo que não ressurjam episódios plenos, podem persistir sintomas que acarretam limitações (Harrow et al., 1990; Gitlin et al., 1995).

Há estudos apresentando evidências de que eventos estressantes ou traumáticos da vida podem precipitar episódios ou interferir na capacidade de recuperação do paciente. Esses eventos podem dizer respeito a problemas familiares (Miklowitz et al., 1988), conjugais (Pardoen et al., 1996), a rupturas de ritmos cronobiológicos (Malkoff-Swchartz et al., 1998), ou serem inerentes ao suporte social e qualidade de relacionamentos afetivos (Ellicot et al., 1990; Stefos et al., 1996; Bauwens et al., 1998).

\section{As intervenções psicossociais}

\section{Psicoeducação}

Embora o termo seja tomado mais facilmente em sentido unidirecional, ou seja, o terapeuta instruindo o paciente e familiares sobre a doença e tratamento, a psicoeducação pode ir além disso. O objetivo, grosso modo, é fazer do paciente um colaborador ativo, aliado dos profissionais de saúde envolvidos e, conseqüentemente, tornar o procedimento terapêutico mais efetivo. Isso é algo mais sofisticado do que a simples transmissão de informações "técnicas", embora existam evidências de que isso, por si, já traga benefícios ao tratamento (Peet e Harvey, 1991; Bauer e McBride, 1996).

A psicoeducação pode ser vista como o estabelecimento de um fluxo de informações de terapeuta para paciente e vice-versa (Callaham e Bauer, 1999). Assim, além de estarem em campo dados objetivos e genéricos sobre o TB e a importância dos medicamen- 
tos, com as vicissitudes de seu uso, poderão ser expostos e analisados outros fatores que interferem na evolução do transtorno e aspectos específicos, próprios do paciente e de seu meio. Por exemplo, é desejável que o paciente explicite seu modo de entender sua situação, reconhecendo ou não a condição mórbida e sua extensão, assim como a necessidade de cuidados sistematizados como o emprego de psicofármacos etc. Tudo isso deve ser abertamente discutido. Também é importante que se conheça a estrutura sociofamiliar e que se possa contar com a ajuda de parentes mais próximos; que se criem condições de reconhecimento precoce dos sintomas e se estabeleçam estratégias de controle, além de se identificarem fatores de vulnerabilidade próprios do paciente.

Para concluir, podemos definir psicoeducação como a tentativa de implementar, no paciente, familiares e profissionais, recursos para lidar com a doença, através do compartilhamento bidirecional de informações relevantes (Callaham e Bauer, 1999). Vale lembrar que pode ser aplicada individualmente, em família ou em diversos tipos de grupos e pode tanto ser combinada com psicoterapia formal como ser aplicada independentemente.

\section{Psicoterapias}

As psicoterapias, às vezes, utilizam técnicas psicoeducativas, mas são normalmente intervenções mais complexas e abrangentes. Seu alvo é aquilo que se constitui um sintoma no universo psíquico humano, incluindo, naturalmente, as conseqüências dele resultantes. O sintoma, aqui, está associado à idéia de patologia, seja em decorrência de uma doença ou transtorno específico, seja como fator de sofrimento emocional de outra origem, mas com a propriedade de prejudicar a saúde mental do indivíduo. Pode ter caráter mais contextual e transitório, geralmente existindo em função da presença de outros sintomas ou de situações críticas e desestabilizadoras, internas, externas ou mistas que se impõem à vida de uma pessoa ou de um grupo; ou ser algo mais estrutural e duradouro, fazendo parte das características do indivíduo. Os sintomas podem ter, então, naturezas e origens diversas, eventualmente difíceis de serem delimitadas com clareza e num mesmo paciente; é possível que se misturem, se superponham e interajam, criando panoramas sindrômicos complexos, tanto para serem compreendidos quanto tratados. Cada tipo de psicoterapia irá considerá-los e tratá-los a partir dos princípios teóricos que a embasam. Algumas tentam aproximar-se de sua origem/causa para compreendê- los e modificá-los ou eliminá-los, como as de orientação psicanalítica, que utilizam o insight e priorizam as noções de psicogênese; outras estão empenhadas em abordá-los de forma mais direta, utilizando técnicas de treinamento de controle e transformação por "correção", como as terapias comportamentais e cognitivas, tendendo a se aterem mais às manifestações sintomáticas em si. As psicoterapias de família e de grupo procuram trabalhar predominantemente na esfera interpsíquica.

As psicoterapias podem funcionar como tratamentos exclusivos (normalmente para transtornos de menor gravidade), ou podem ser associadas a tratamentos farmacológicos para facilitá-los e complementá-los, na medida que eles sozinhos não sejam suficientes para tratar de todos os sintomas inerentes ou coexistentes ao transtorno mais grave como, por exemplo, о ТВ. Há autores que sugerem, inclusive, que seriam apropriados diferentes tipos de intervenção, dependendo do estágio da doença, no caso do TB (Rothbaum e Astin, 2000).

As intervenções psicossociais para o TB

Em princípio, várias modalidades de intervenções psicossociais podem ser utilizadas no TB: processos individuais, familiares e grupais; psicoterápicos, especificamente psicoeducativos, ou combinados. São exemplos: as psicoterapias derivadas da psicanálise (transposições da teoria psicanalítica para intervenções geralmente mais breves, focais e dirigidas à modificação de sintomas específicos), psicoterapia interpessoal, psicoterapia cognitivo-comportamental, psicoterapia interpessoal e de ritmos sociais, as terapias de família, incluindo as de casal, e as intervenções de grupo, que podem ser de diversas diretrizes teóricas (Scott, 1995; Bauer et al., 1998; Colom et al., 1998; Rothbaum e Astin, 2000; Huxley et al., 2000).

Os estudos disponíveis na literatura sugerem que as intervenções psicossociais são úteis no tratamento do TB, mas, como é apontado por muitas das revisões mais recentes, as deficiências metodológicas da maioria desses trabalhos não permitem a afirmação segura da eficácia de tais intervenções terapêuticas dentro dos padrões de exigência da ciência na atualidade (Scott, 1995; Colom et al., 1998; Huxley et al., 2000). Os estudos têm sido freqüentemente realizados sem respeitar critérios necessários para a produção de informações científicas de boa qualidade, como, por exemplo, o emprego de grupos de controle, rando- 
mização, avaliação cega dos resultados, uso de métodos terapêuticos claramente definidos e padronizados através de manuais e definição de metas clinicamente relevantes e objetivamente verificáveis. Contudo, têm sido publicados, mais recentemente, estudos de boa qualidade metodológica e que demonstram a eficácia de diversas modalidades de intervenções psicossociais, especialmente as que incluem métodos psicoeducativos.

Uma revisão (Callaham e Bauer, 1999) cita quatro formas de psicoterapias estruturadas aplicadas com base em manuais e especificamente elaboradas para $o$ TB. As quatro formas têm em comum alguns aspectos, como: a) partirem do fato de que o TB é, em grande parte, biologicamente determinado, embora sofra influência do ambiente; b) serem administradas como coadjuvantes à farmacoterapia; e c) buscarem aumentar o nível de adesão à farmacoterapia. Essas intervenções são brevemente descritas a seguir.

A Terapia Interpessoal e de Ritmos Sociais (Frank et al., 1994; 2000) é aplicada individualmente e foi desenvolvida especificamente para o tratamento do TB a partir da hipótese do envolvimento de alterações de ordem cronobiológica na gênese do transtorno. Há a pressuposição de um distúrbio genético que induziria as anormalidades do ritmo circadiano e do ciclo sono-vigília que, por sua vez, estariam relacionados aos sintomas bipolares. Os eventos da vida, tanto negativos quanto positivos, poderiam ser responsáveis por rupturas da regularidade cronobiológica e a terapia, combinando princípios de psicoterapia interpessoal e técnicas comportamentais, procura preservar essa regularidade. Está em andamento um estudo controlado, randomizado, visando principalmente à avaliação da eficácia desse tipo de intervenção no TB I, realizado por Ellen Frank et al., da Universidade de Pittsburg, na Pensilvânia. Apesar de não estarem ainda publicados resultados mais definitivos, o grupo já divulgou dados interessantes obtidos nessa pesquisa como, por exemplo, a verificação de que é possível induzir a regularização no estilo de vida de pacientes bipolares através dessa modalidade de intervenção (Frank et al., 1997). Outro achado que parece reforçar a idéia do papel protetor da regularização dos ritmos sociais/circadianos foi a constatação de que, ao trocarem a modalidade de tratamento do experimental para o tratamento-controle (Manejo Clínico Intensivo) e vice-versa, os pacientes apresentaram maiores índices de recaída e maior persistência sintomática do que aqueles que permaneceram no mesmo tratamento, independentemente de qual fosse ele, embora os autores afirmem que a interpretação desses resultados ainda mereça cuidado (Frank et al., 1999).

O Programa de Metas de Vida (Life Goals Program) foi elaborado por Mark Bauer e Linda McBride, os quais, após vários anos de trabalho com pacientes bipolares, identificaram quatro temas principais em torno do tratamento desses pacientes: 1) a baixa qualidade de vida se a doença não estiver sob controle; 2) os pacientes freqüentemente não se engajam adequadamente no processo terapêutico como um todo; 3) pessoas acometidas por TB manifestam limitações funcionais (sociais e ocupacionais) significativas, mesmo após os sintomas agudos do TB estarem sob controle; 4) os projetos de melhoria funcional raramente são discutidos com o paciente. Assim, criaram uma modalidade de terapia de grupo, dando ênfase a esses aspectos. Ela foi estruturada para ser realizada em duas fases distintas, nas quais os terapeutas seguem os padrões de um manual. A primeira fase consiste de cinco sessões psicoeducativas, e o objetivo é que os pacientes conheçam sua doença e modos de tratá-la, para que possam adquirir capacidade de lidar com seus sintomas e agir em máxima colaboração com seus médicos. A segunda fase é centrada na melhora do funcionamento social e ocupacional, através de metas definidas junto ao paciente. Nessa, não há número de sessões pré-definido, pois isso depende do tempo necessário para alcançar uma ou mais metas, e podem ser empregadas técnicas oriundas da psicoterapia interpessoal ou cognitivocomportamental (Bauer e McBride, 1996).

O Tratamento Focado na Família (Miklowitz et al., 2000) ressalta a influência que o meio familiar pode exercer no curso dos vários transtornos psiquiátricos, entre eles, o TB. A terapia é programada para ocorrer em três módulos, envolvendo o paciente e sua família (o que pode significar pais, cônjuges, irmãos, filhos ou outras pessoas que vivam próximas a ele) e, em pelo menos um dos estudos feitos pelo grupo, as sessões foram realizadas nas próprias residências dos pacientes. O primeiro módulo prevê sete sessões, essencialmente de caráter psicoeducativo, em que paciente e família são amplamente informados sobre as características da doença; é enfatizada a importância da adesão ao tratamento e tenta-se identificar peculiaridades do caso em questão, assim como planejar estratégias de manejo. No segundo módulo, durante sete a dez sessões, todo o grupo familiar aprende a lidar com o estresse gerado por problemas de comunicação, desenvolvendo habilidades para modificar padrões a eles relacionados. Finalmente, no terceiro módulo, em qua- 
tro ou cinco sessões, aprendem a identificar problemas que surgem no período pós-episódico (por exemplo, retorno ao trabalho), e a criar soluções para eles. Nesse tipo de terapia, está muito presente a idéia de Emoção Expressa (Expressed Emotion), que é a manifestação emocional, dentro da família, geralmente de tons críticos ou hostis e que, potencialmente, representa fator de risco de precipitação ou agravamento de crises para o paciente (Miklovitz et al., 1995; 2000).

Outra intervenção psicossocial, especificamente adaptada para o TB, é uma combinação especial de métodos clássicos de Terapia Cognitivo-Comportamental e elementos psicoeducativos, idealizada por Basco e Rush (1996) e cujo objetivo primário é a máxima adesão ao tratamento e a prevenção de recaídas (Callahm e Bauer, 1999).

Ademais, vale a pena citar alguns estudos com maiores ou menores afinidades com os métodos mencionados acima realizados por diferentes pesquisadores. Entre esses, há um estudo controlado e randomizado, em que os autores avaliaram a eficácia da prática de se ensinar o paciente com TB a identificar sintomas precoces de recorrência de episódios agudos, considerados idiossincráticos pelos autores, e prepará-lo a procurar ajuda imediata para o auxílio no controle da doença. Foram realizadas de sete a doze sessões individuais com o intuito de esclarecer e treinar o paciente, associadas ao tratamento medicamentoso; o grupo-controle recebeu somente o tratamento medicamentoso e de suporte-padrão. Os resultados sugeriram que o método ajudou a espaçar as recorrências de episódios maníacos, não mostrou efeitos equivalentes para prevenir novos quadros depressivos e contribuiu para melhorar a performance social e laborativa (Perry et al., 1999). Em outro ensaio (Zaretsky et al., 1999), foram comparados os resultados entre um grupo de 11 pacientes com depressão bipolar e outro de também 11 pacientes com depressão unipolar, recebendo 20 sessões em regime semanal de terapia baseada no protocolo-padrão elaborado por Basco e Rush (1996) já mencionado, associadas a estabilizadores do humor no primeiro caso, e como tratamento exclusivo, no segundo. A remissão dos sintomas depressivos foi semelhante nos dois grupos, mas atitudes disfuncionais subjacentes, nos bipolares, não responderam tão bem, o que levou os autores a considerar que, possivelmente, esses pacientes necessitem de maior intensidade na administração desse tipo de psicoterapia. Merece também atenção um estudo aberto, feito no Canadá (Patelis-Siotis et al., 2001), sobre o uso de grupoterapia baseada em princípios cognitivo-comportamentais e aplicada mediante uso de manual. Foi constatada melhora significativa no funcionamento social dos pacientes estudados após 14 sessões semanais de tratamento, em uso de estabilizadores do humor e já fora do período agudo no início da psicoterapia. Em outro estudo (Davemport et al., 1977), foram avaliados os efeitos da psicoterapia aplicada a grupos de casais de pacientes com TB, em uso concomitante de lítio, e comparados com controles tratados só com lítio. Foi observado, num acompanhamento entre dois e dez anos após a alta de uma internação/referência, que os pacientes recebendo a terapia de casais tiveram menor número de hospitalizações, menor índice de rupturas matrimoniais e melhor desempenho no funcionamento social. Na Universidade de Stanford, EUA, dois estudos (Volkmar et al., 1981; Shakir et al., 1979) demonstraram os efeitos benéficos do uso de psicoterapia de grupo, no formato interpessoal/interacional descrito por Yalom (Yalom, 1975), quanto à queda acentuada no número de hospitalizações e aumento do índice de emprego, comparando os resultados do grupo em terapia por dois anos com os dados do mesmo grupo nos dois anos que precederam o início da intervenção. Os pacientes faziam uso de lítio e estavam estabilizados ao iniciar o estudo e continuaram a tomar regularmente o medicamento durante o experimento.

Vários estudos feitos com famílias, além dos de Miklovitz (2000), apontam para a eficácia, seja de intervenções psicoeducativas somente (Honig et al., 1997), ou de psicoterapias propriamente ditas, administradas ao grupo familiar. Num estudo multicêntrico (Glick et al., 1994), em que escalas de avaliação foram aplicadas a pacientes, familiares e médicos, foi descrita uma melhoria na resolução de episódios agudos e de funcionamento global em pacientes que, eventualmente, com suas famílias, receberam intervenções psicoeducativas, mesmo de forma não-sistematizada, no contexto do tratamento medicamentoso.

Um estudo recente (Colom et al., 2003) avaliando 120 pacientes com TB I e II em remissão por pelo menos seis meses, emparelhados por sexo e idade, randomizados, para receberem, além do tratamento medicamentoso, 21 sessões psicoeducativas formais em grupo ou 21 sessões de encontros em grupo sem intervenções estruturadas, demonstrou que o método de psicoeducação foi eficaz para prevenir recaídas. Outro estudo (Lam et al., 2003) controlado e randomizado, com 103 pacientes, utilizou terapia cognitiva em grupo para avaliar eficácia para prevenção de recaída e apresentou resultados positivos, como 
diminuição do número de episódios, número de dias de duração dos episódios, taxa de internação e melhora do funcionamento social e da capacidade de lidar com pródromos da mania durante os 12 meses de duração do tratamento. Também lançando mão de metodologia cuidadosa, outro estudo (Miklowitz et al., 2003) controlado e randomizado, com 101 pacientes em uso de medicação, com duração de dois anos e comparando terapia familiar (que incluiu método psicoeducativo) com intervenção-padrão para controle de crises, concluiu que o tratamento familiar psicoeducativo melhorou a adesão medicamentosa e o ajustamento pós-episódico.

\section{Comentários}

Quando tentamos precisar o papel das intervenções psicossociais no panorama dos procedimentos terapêuticos para o TB, nos deparamos com uma situação que, curiosamente, vem se mantendo por mais tempo do que seria de se esperar: a maioria dos estudos aponta para a utilidade dessas intervenções, mas sem dados suficientemente consistentes para afirmar inequivocamente sua eficácia e efetividade. Isso acontece basicamente pela razão já aludida antes neste trabalho, das falhas metodológicas nos desenhos dos experimentos. Também pouco se tem feito para verificar se há maior adequação no uso de certas modalidades psicoterápicas em relação a outras, embora alguns autores emitam opiniões a respeito (Colom et al., 2001). Assim, há carência de evidências científicas que demonstrem como e quanto agem os diferentes tipos de psicoterapias e métodos psicoeducativos sobre o TB. As revisões têm acentuado insistentemente essas questões e demandado novos estudos com desenhos mais adequados. Alguns pontos relevantes citados a título de demanda para possíveis futuros estudos são, por exemplo: padronização de métodos de diagnóstico e medição de sintomas, verificação da relação entre o tipo de intervenção e o modo de apresentação da doença em que a eficácia se torna evidente, a necessidade de se considerar a complexidade do TB no que tange aos diferentes aspectos para os quais as intervenções psicossociais devem estar endereçadas, os estudos devem ter desenhos científicamente apropriados e também que possíveis intervenções que pretendam ser específicas para o TB se baseiem em modelos teóricos claros sobre o que seria o TB (Jones, 2003). Quanto aos modos através dos quais uma intervenção psicossocial poderia operar terapeuticamen- te no TB, diversas possibilidades têm sido aventadas, como a atuação sobre sintomas subsindrômicos entre os episódios, manejo de comorbidades no campo dos transtornos da personalidade, melhora da adesão medicamentosa, redução de insônia e irregularidades no ritmo circadiano, diminuição do impacto de fatores estressantes, para mencionar algumas (Zaretsky, 2003). No presente, estão sendo publicados trabalhos mostrando que a situação está se modificando e que, possivelmente, em futuro próximo, teremos dados confiáveis em número suficiente para tirarmos conclusões mais robustas.

Numa revisão já citada (Scott, 1995), o autor reflete sobre a carência de dados no campo em questão e presume que as abordagens psicossociais tenham sido um tanto quanto negligenciadas para o $\mathrm{TB}$ por motivos tais como a predominante importância dada aos fatores biológicos na etiologia, a crença na recuperação interepisódica mais completa do que o é na realidade e, por fim, o papel desestimulador de alguns psicanalistas quando tentaram tratar psicoterapicamente indivíduos com sintomas maníacos. É provável que existam mais fatores a serem considerados, pelo menos no que diz respeito às dificuldades de se estudarem adequadamente as intervenções psicossociais. Em princípio, poderia ser lembrada a complexidade e quase inextrincável subjetividade que caracteriza o universo das psicoterapias. Elas foram criadas e desenvolvidas a partir de sistemas de pensamento diferentes dos que hoje predominam, quando se fala de ciência. Na virada do século XIX para o XX e nas primeiras décadas deste, quando a psicologia começou a adquirir maior importância e a psicanálise apareceu, ganhava força, através de Edmund Husserl, Franz Brentano e Max Weber, entre outros, a idéia de que o homem deveria ser estudado em separado das ciências da natureza, ou seja, enquanto às ocorrências naturais deveriam corresponder leis causais, mais diretas, o ser humano deveria ser interpretado e compreendido de modo sem contrapartida na natureza (Watson, 2002). Essa idéia parece ter sido muito penetrante em grande parte das tentativas feitas no sentido de se compreender e interferir no comportamento humano. Tanto as psicoterapias que se originaram diretamente da psicologia quanto as que surgiram a partir da psicanálise sustentam-se mais através de construtos de coerência teórica intrínseca e práticas caracterizadas pela singularidade do que por fundamentos estabelecidos prioritariamente pela experiência empírica, verificáveis objetiva e sistematicamente. Portanto, não 
há um encaixe exato ao modelo médico mais difundido, que é ajustado à ciência da natureza. Existem diferenças entre os diversos tipos de psicoterapias e entre os princípios que as orientam, mas não o suficiente para isentar uma ou mais delas inteiramente das dificuldades inerentes ao tema de que estamos tratando. Então, pode-se antever que, dentro das tendências da pesquisa atual, empreender estudos realmente elucidativos sobre as psicoterapias é algo que exige flexibilidade e algum esforço extra por parte dos psicoterapeutas, principalmente no que diz respeito ao ajuste entre as condições de satisfação de critérios científicos e a exeqüibilidade do processo terapêutico, sem que ele seja descaracterizado. As intervenções de cunho unicamente psicoeducativo, por serem mais restritas e objetivas, são mais fáceis de serem aplicadas e avaliadas.

Para ilustrar parcialmente o ponto de vista acima, podemos falar da difícil e necessária tarefa de se aplicar um tratamento de forma padronizada, quando ele é fortemente marcado pela intersubjetividade e imprevisibilidade da relação terapeuta/paciente. É mesmo válido dizer que essa relação entre os dois sujeitos torna-se instrumento da terapia e que precisa ser manejada com cuidado para se evitar as estereotipagens e o esmaecimento das identidades. A necessidade de se estruturar o procedimento psicoterápico que se quer estudar pode ser encarada como um entrave ou mesmo algo incompatível com a natureza de uma psicoterapia, pois a maioria dos terapeutas tende a ver com desconfiança as práticas baseadas em manuais ou instrumentos semelhantes. Possivelmente, isso gera um certo desânimo nos profissionais quando desejam submeter uma modalidade de intervenção terapêutica à pesquisa, norteada pelas exigências do modelo científico ao qual nos referimos, segundo o qual torna-se importante uniformizar procedimentos para que sejam passíveis de apreciação objetiva e reprodutibilidade. Outro aspecto é o papel que normalmente representam as entrevistas iniciais nas psicoterapias. Também, quanto à verificação dos efeitos da psicoterapia sobre os indivíduos, é fundamental minimizar a sombra da subjetividade, através de critérios bem definidos sobre os resultados e as maneiras de verificá-los. Estes aspectos, que sabemos não serem de resolução tão simples quanto gostaríamos e que com certeza não esgotam o problema, parecem ser, contudo, de abordagem inevitável para a elaboração de projetos mais rigorosos e a obtenção de respostas confiáveis ao grande número de interrogações sobre as intervenções psicossociais para o TB. Em outras áreas diagnósticas, como da esquizofrenia e mesmo da depressão unipolar, a pesquisa a respeito da eficácia da psicoeducação e das psicoterapias como tratamentos complementares ao farmacológico está mais avançada.

\section{Conclusão}

Atualmente, tornou-se imprescindível demonstrar cientificamente a eficácia e mesmo a efetividade de qualquer tratamento. As intervenções psicossociais, de modo geral, ainda carecem de estudos metodologicamente adequados, suficientes para serem reconhecidas e preconizadas com maior propriedade. Há vários trabalhos salientando a sua utilidade, mas, apesar de vários estudos com desenho mais rigoroso terem sido recentemente publicados, ainda não há dados que possam sustentar com clareza e robustez o que até agora tem sido apresentado de maneira mais impressionista. É importante identificar problemas que possam dificultar os progressos nesse sentido e encontrar soluções que os contornem. As psicoterapias, comumente, caracterizam-se pela subjetividade tanto de operação quanto de avaliação de resultados. Esses aspectos, possivelmente, são importantes enquanto fatores geradores de entrave à realização de avaliações necessárias. Mesmo levando-se em conta a complexidade da questão, há de se buscar criar condições que combinem exeqüibilidade do processo terapêutico sem degenerá-lo e estruturação/ padronização de aplicação, assim como verificação objetiva e clara de seus efeitos. 


\section{Referências bibliográficas}

AKISKAL, H.S. - The Prevalent Clinical Spectrum of Bipolar Disorders: Beyond DSM-IV. J Clin Psychopharmacol 16 suppl: 4-14, 1996.

Akiskal, H.S.; PINTo O. - The Evolving Bipolar Spectrum. The Psychiatric Clinics of North America 22: 517-34, 1999.

American Psychiatric Association. - Practice Guideline for the Treatment of Patients with Bipolar Disorder (Revision). Am J Psychiatry 159 (suppl 4): $1-50,2002$

Basco, M.R.; Rush, A.J. - Cognitive Behavioral Therapy for Bipolar Disorder. The Guilfor Press, New York.

Bauer, M.; McBride, L. - Structured Group Psychotherapy for Bipolar Disorder: The Life Goals Program. Springer, New York, 1996.

Bauer, M.; McBride, L.; Chase, C.; Sachs, G.; SheA, N. - Manual Based Group Psychotherapy for Bipolar Disorder: A Feasibility Study. J Clin Psychiatry: 59-9; 449-55, 1998.

Baumens, F.; Pardoen, D.; Staner, L.; Dramaix, M.; Mendlewicz, J. - Social Adjustment and the Course of Affective Illness: a One Year Controlled Longitudinal Study Involving Bipolar and Unipolar Outpatients. Depression and Anxiety: 8;50-7, 1998.

Callaham, M.A.; Bauer, M.S. - Psychosocial Interventions for Bipolar Disorder. The Psychiatric Clinics of North America 22: 675-88, 1999.

Classificaçāo de transtornos mentais e de comportamento da CiD-10. Artes Médicas, Porto Alegre, 1993

Colom, F.; Vieta, E.; Martinez-Aran, A.; Reinares, M.; Goikolea, J.; Benabarre, A.; Torrent, C. Comes, M. Corbella, B. Parramon, G. Corominas, J. A. - A Randomized Trial on the Efficacy of Group Psychoeducation in the Prophilaxis of Recurrences in Bipolar Patients whose Disease is in Remission. Arch Gen Psychiatry 60: 402-71, 2003.

Colom, F.; Vieta, E.; Martinez, A.; Jorouera, A.; Gastó, C. - What is the Role of Psychotherapy in Treatment of Bipolar Disorder?. Psychother Psychosom 67: 3-9, 1998.

Colom, F.; Martinez-Arán, A.;Vieta, E. - Ineficacia de la Psicoterapia en los Transtornos Bipolares: Breve Revision Com Un Final Feliz; In Transtornos Bipolares/Avances Clínicos y Terapéuticos, Médica Panamericana, Madrid: 297-311, 2001.

Davenport, Y.; Ebert, M.; Adland, M.; Goodwin, F. - Couples Group Therapy as an Adjunct to Lithium Maintenance of the Manic Patient. Amer J Orthopsychiatry 473: 495-502, 1977.

Dion, G.L.; Tohen, M.; Anthony, W. - Symptoms and Functioning of Patients with Bipolar Disorder Six Months after Hospitalization. Hospital and Community Psychiatry 39: 652-7, 1988.

Dominic, L.; Watkins, E.; Hayward, P., Bright, J., Wright, K.; Kerr, N.; ParrDavis, G.; Sham, P. - A Randomized Controlled Study of Cognitive Therapy for Relapse Prevention for Bipolar Disorder. Arch Gen Psychiatry 60: 145-52, 2003.

Ellicott, A.; Hammen, C.; Gitlin, M.; Brown, G.; Jamison, K. - Life Events and the Course of Bipolar Disorder. Am J Psychiatry 147: 1194-8, 1990.

Frank, E., Hlastala, S., Ritenour, A., Houck, P.; Tu, X.; Monk, T.; Mallinger, A.; KUPFER, D. - Inducing Lifestyle Regularity in Recovering Bipolar Disorder Patients: Results from the Maintenance Therapies in Bipolar Disorder Protocol. Biol Psychiatry 41: 1165-73, 1997.

Frank, E.; Ehlers, C.; Monk, T.; Cornes, C.; Carter, S.; Frankel, D. - Interpersonal and Social Rhythm Therapy for Bipolar Disorder: Integrating Interpersonal and Behavioral Approaches. The Behavior Therapist 17: 143-9, 1994.

Frank, E.; Swartz, H.; KuPfer, D. - Interpersonal and Social Rhythm Therapy: Managing the Chaos of Bipolar Disorder. Biol Psychiatry 48: 593$604,2000$.

Frank, E.; Swartz, H.; Mallinger, A.; Thase, M.; Weaver, E.; Kupfer, D. - Adjunctive Psychotherapy for Bipolar Disorder: Effects of Changing Treatment Modality. Journal of Abnormal Psychology 108: 579-87, 1999.

Fromm Reichmann, F. - Intensive Psychotherapy of Manic-Depressives. Confínia Neurológica 9: 158-65, 1949.

Gituin, M.J.; Swendsen, J.; Heller, T.L. ET AL. - Relapse and Impairment in Bipolar Disorder. Am J Psychiatry 152: 1635-40, 1995.

Glick, I.; Burti, L.; Okonogi, K.; SAcks, M. - Effectiveness in Psychiatric Care Psychoeducation and Outcome for Patients with Major Affective Disorder and their Families. British Journal of Psychiatry 164: 104-6, 1994.

Goodwin, F. K.; JAmison, K. R. - Manic-Depressive Illness. New York, Oxford University Press, 1990
Guze, S.B.; Robins, E. - Suicide and Primary Affective Disorders. British Journal of Psychiatry 117: 437-8, 1970.

Harrow, M.; Goldberg, J.; Grossman, L.; Meltzer, H. - Outcome in Manic Disorders. Arch Gen Psychiatry 47: 665-71, 1990.

Honig, A.; Hofman, A.; RozendaAl, N.; Dingemans, P. - Psychoeducation in Bipolar Disorder: Effect on Expressed Emotion. Psychiatry Research 72: 17-22, 1997

Huxley, N., Parikh, S.; Baldessarini, R. - Effectiveness of Psychosocial Treatments in Bipolar Disorder: State of Evidence. Harvard Ver Psychiatry 8: 126-40, 2000.

Jones, S. - Psychotherapy of Bipolar Disorder: a Review. Journal of Affective Disorders; article in press in 2003.

Kusumakar, V.; Yathan, L.; Haslam, D.; Parikh, S.; Matte, R.; Sharma, V.; Silverstone, P.; Kutcher, S.; Kennedy, S. - The Foundations of Effective Management of Bipolar Disorder. Can J Psychiatry 42 (suppl): 69s-73s, 1997.

Malkoff-Schwartz, S.; Frank, E.; Anderson, B.; Sherril, J.; Siegel, L.; Patterson, D.; KuPFER, D. - Stressful Life Events and Social Rhythm Disruption in the Onset of Manic and Depressive Bipolar Episodes. Arch Gen Psychiatry 55: 702-7, 1998.

Miklowitz, D.; Goldstein, M.; Nuechterlein, K. - Verbal Interactions in the Families of Schizophrenic and Bipolar Affective Patients. Journal of Abnormal Psychology;104;268-76, 1995.

Miklowitz, D.; Goldstein, M.; Naechterlein, K.; Snyder, K.; Mintz, J. - Family Factors and the Course of Bipolar Affective Disorder. Arch Gen Psychiatry 45: 225-31, 1988.

Mirlowitz, D.; Simoneau, T.; George, E.; Richards, J.; Kalbag, A.; Sachs-Ericsson, N.; Suddath R. - Family-Focused Treatment of Bipolar Disorder: 1Year Effects of a Psychoeducational Program in Conjunction with Pharmachotherapy. Biol Psychiatry 48: 582-92, 2000.

Miklowitz, D.; George, E.; Richards, J.; Simoneau, T.; Suddath, R. - A Randomized Study of Family-Focused Psychoeducation and Pharmachotherapy in the Outpatient Management of Bipolar Disorder. Arch Gen Psychiatry 60: 904-12, 2003.

Muller-Oerlinghausen, B. - Does Effective Lithium Prophilaxis Result in a Symptom-Free State of Manic-Depressive Illness? Some Thoughts on the Fine-Tuning of Mood Stabilization. Comprehensive Psychiatry 41 (supl.1): 26-31, 2000.

Pardoen, D; Baumens, F; Dramaix, M.; Tracy, A.; Genevrois, C.; Staner, L.; Mendlewicz, J - Life Events and Primary Affective Disorders a One Year Prospective Study. British Journal of Psychiatry 169: 160-6, 1996.

Patelis-Siotis, I.; Young, L.; Robb, J.; Marriott, M.; Bieling, P.; Cox, L.; Joffe, R. Group Cognitive Behavioral Therapy for Bipolar Disorder: a Feasibility and Effectiveness Study. Journal of Afective Disorders 65: 145-63, 2001.

Peet, M; Harvey, N. - Lithium Maintenance: a Standard Education Programme for Patients. British Journal of Psychiatry 158: 197-200, 1991.

Perry, A.; TARrier, N.; Moriss, R.; McCarthy, E.; Limb, K. - Randomised Controlled Trial of Efficacy of Teaching Patients with Bipolar Disorder to identify Early Symptoms of Relapse and Obtain Treatment. BMJ 318: 149-53, 1999.

Rothbaum, B.; Astin, M. - Integration of Pharmachotherapy and Psychotherapy for Bipolar Disorder. J Clin Psychiatry 61 (suppl 9): 68-75, 2000.

Scotт, J. - Psychotherapy for Bipolar Disorder. British Journal of Psychiatry 167: 581-8; 1995.

Shakir, S.; Volkmar, F.; Bacon, S.; Pfefferbaum, A. - Group Psychotherapy as an Adjunct to Lithium Maintenance. Am J Psychiatry 136: 455-6, 1979.

Stefos, G.; Bauwens, F.; Staner, L.; Pardoen, D.; Mendlewicz, J. - Psychosocial Predictors of Major Affective Recurrences in Bipolar Disorder: a 4Year Longitudinal Study of Patients on Prophylactic Treatment. Acta Psychiatr Scand 93: 420-6, 1996.

Volkmar, F.; Bacon, S.; Shakir, S.; Pfefferbaum A. - Group Therapy in the Management of Manic-Depressive Illness. Am JPsychoth 35: 226-34, 1981.

Watson, P. - The Modern Mind. Perennial, New York; 46, 2002.

YALOM, I. D. - The Theory and Practice of Group Psychotherapy. Basic Books, New York, 1975.

Zaretsky, A.; Segal, Z.; Gemar, M. - Cognitive Therapy for Bipolar Depression: a Pilot Study. Can J Psychiatry 44: 491-4, 1999.

ZARETSKY, A. - Targeted Psychosocial Interventions for Bipolar Disorder. Bipolar Disord 5 (suppl 2): 80-7, 2003. 